\title{
Effect of Trema guineensis leaves (celtidaceae) on glucose-induced hypertension in Wistar rats
}

\author{
Balakiyém Kadissoli ${ }^{1 *}$, P A Mouzou ${ }^{1}$, T Pakoussi ${ }^{1}$, K Eklu-Gadegbeku ${ }^{1}$, A K Aklikokou ${ }^{1,2}$, M Gbeassor $^{1,2}$ \\ ${ }^{1}$ Laboratoire de Physiologie/Pharmacologie des Substances Naturelles, Faculté des Sciences, Université de Lomé-Togo, Lome, Togo, ${ }^{2}$ Department of Animal \\ Physiology and Pharmacology, Centre de Recherche et de Formation sur les Plantes Médicinales, Université de Lomé-Togo, Lomé, Togo
}

\section{ARTICLE INFO \\ Article history: \\ Received on: May 23, 2018 \\ Accepted on: September 29, 2018 \\ Available online: January 24, 2019}

Key words:

Antioxidant,

Glucose,

Hypertension,

Trema gunieensis,

Wister rat.

\begin{abstract}
Trema guineensis' leaves are used in Togolese's traditional medicine as an antihypertensive remedy. This study aims at evaluating the protective's effect of T. guineensis' extract on glucose-induced hypertension to Wistar rats. Hypertension has been induced to rats through oral administration of $10 \% \mathrm{D}$-glucose for 3 weeks. Animals have received the treatments for 3 weeks. At the end of the treatments, blood pressure, reduced glutathione (GSH), malondialdehyde (MDA), superoxide dismutase (SOD), nitric oxide (NO) activity in heart, aorta, liver, and kidney and biochemical parameters in serum have been measured. At the doses of 200 and $400 \mathrm{mg} / \mathrm{kg} / \mathrm{day}$, T. guineensis has significantly prevented the increase in high blood pressure in glucose's presence. The extract has significantly prevented the high concentrations of total cholesterol, and triglycerides, low-density lipoprotein (LDL), atherogenic index, urea, and uric acid induced by glucose treatment as compared to untreated glucose's rats group. T. guineensis $(400 \mathrm{mg} / \mathrm{kg}$ ) has prevented the increase in glutamic-oxaloacetic transaminase, GOT but did not affect serum creatinine's rates. Treatment with plant's extract has significantly prevented the increase in SOD, MDA, and the decrease in GSH and NO's concentration in aorta, heart, kidney, and liver. These results have proved that the extracted leaves of $T$. guineensis could prevent glucose-induced hypertension. These results have allowed the traditional use of $T$. guineensis.
\end{abstract}

\section{INTRODUCTION}

Hypertension is one of the most wide-spread and modifiable factors of cardiovascular diseases throughout the world $[1,2]$. It is also one of the main causes of disability, morbidity, and mortality [3]. From far away, it has been the most common affection among cardiovascular diseases, and it is considered as a serious problem of public health's matter. Indeed, there is about 80 million of hypertensive in Sub-Saharan Africa, and the projections based on epidemiological data suggest that this figure will reach 150 million patients before 2025 [4]. Among the 17 million patients who die every year from cardiovascular diseases, it is thought that 7-8 million are hypertensive [4]. African countries are particularly concerned because they, in fact, think that in 2025 almost $3 / 4$ of the hypertensive world's population will live in developing countries. Despite the availability of several antihypertensive agents, who successfully reduce blood pressure in many hypertensive subjects, numerous patients remain unresponsive to treatment and are left with high blood pressure [2]. For example, hypertension is not adequately managed in approximately $10 \%$ of patients who are compliant with

*Corresponding Author:

Balakiyém Kadissoli,

Laboratoire de Physiologie/Pharmacologie des Substances Naturelles, Faculté des Sciences, Université de Lomé-Togo. PO. Box 2936.

Email: kadissoliarmiss@gmail.com prescriptive therapeutics [5]. Existing review has shown that in many developing countries, about $70-80 \%$ of the population, have relied arduously on medicinal plants in their vicinity to meet their basic health-care needs [6].

Fructose and glucose are two carbohydrates source blood pressure's increased $[7,8]$. Several mechanisms are the source of this blood pressure's increase, of which the most important are dyslipidemia [9], sodium retention and fluid volume expansion [10], stimulation of the sympathetic nervous system [11], endothelial dysfunction [12], and also the increase in oxidative stress [13].

Some plants are used to treat hypertension by traditional healers in Africa. Among them, Trema guineensis is a plant commonly used in traditional medicine to treat several pathologies. The leaves' decoction of $T$. guineensis is drunk in case of high blood pressure associating it with Gardenia ternifolia leaves [14]. Wooden fragments are roasted and used to make the tea which fights against dysentery; the bark is used for cough's treatment. Bark's decoction is used against bronchial congestion, asthma, sore throat, teeth, and against mouth's infections. Our previous studies have shown that the extract of $T$. guineensis leaves had vasodilator effects [15].

Therefore, the present study has been conceived to investigate on antihypertensive activities of $T$. guineensis on a rat model of glucoseinduced hypertension. 


\section{MATERIALS AND METHODS}

\subsection{Plant Material and Extraction}

Fresh pieces of $T$. guineensis' leaves have been harvested in December 2016 in Badou, a village located at $220 \mathrm{Km}$ from Lomé (Togo) and authenticated by the Department of Botany and Plant Ecology in Science Faculty (University of Lomé) where voucher specimen (N 15379TG) was deposited. Leaves are washed in the tap water then dried in light's shelter. Once these leaves are dried, they are reduced in powder evaluated at $250 \mathrm{~g}$. This quantity was mortified by uninterrupted agitation during $72 \mathrm{~h}$ in an extraction' solvent composed of water and ethanol in a voluminal ratio 20:80. After soaking, the product was first filtered on the cotton wool, and after, on the filter paper and then, the filtrate evaporated in a rotavapor R-210 (Buchi) at $45^{\circ} \mathrm{C}$. We obtained $28.2 \mathrm{~g}$ of extract which corresponds to $11.28 \%$.

\subsection{Phytochemical Screening}

Phytochemical analyses of the ethanolic extract have been done following the procedure described by Odebiyi and Sofowora [16]. The plant's extract was screened in the presence of alkaloids, saponins, tannins, and flavonoids. The total phenols were determined from the Folin-Ciocalteu method after polyvinylpolypyrrolidone tannin fixation. The determination of total flavonoids is based on flavonoids' property to form with aluminum chloride aluminum chelates of Mimica-Dukic.

\subsection{Animals}

Albinos Wistar rats, weighting between 150 and 180 g, have been used. Animals were raised in the Animal House of Science Faculty (University of Lomé) in plastic cages, under standard light (12-h day/night natural cycle) and temperature $\left(25^{\circ} \mathrm{C}\right)$. Rats were fed with standard diet and water ad libitum. The research protocol was approved by the Institutional Animal Ethical committee with proposal number: 018/2017/CB-FDS-UL Date: 01/10/2017.

\subsection{Experimental Design}

The extract's effect of T. guineensis on D-glucose-induced hypertension has been evaluated. Normotensive rats were randomly divided into five groups composed of five animals each which have received different treatments by gavage. Group one has received tap water and has served as negative control (NC), group two has received 10\% D-glucose solution in drinking water serving as positive control (PC). Groups III and IV were the treated groups which have received each $10 \% \mathrm{D}$-glucose in drinking water and the plant's extract by gavage at $200 \mathrm{mg} / \mathrm{kg} /$ day and $400 \mathrm{mg} / \mathrm{kg} /$ day, respectively. Group five has received D-glucose in drinking water and nifedipine at $10 \mathrm{mg} / \mathrm{Kg}$ by gavage. The experiments have lasted 3 weeks. At the end of the experiment, systolic arterial blood pressure rate was measured as described by Mtopi et al. [17]. After blood pressure measurement, blood was collected at the level of the retro-orbital sinus. Serum was separated and total cholesterol, highdensity lipoprotein (HDL)-cholesterol, triglycerides, urea, glutamicoxaloacetic transaminase (GOT), and GTP rates using commercial diagnostic kits BC 3000 + Mindray. After blood collection, heart, aorta, liver, and kidney were removed, weighted, crushed, and homogenized in McEwen solution for aorta and heart or Tris- $\mathrm{HCl}$ buffer solution for liver and kidney. Serum and homogenate were stored at $-20^{\circ} \mathrm{C}$. Reduced glutathione (GSH) was determined by the method of Sedlak and Lindsay [18]. Superoxide dismutase (SOD) was determined using the method of Misra and Fridovich [19]. Nitric oxide (NO) was determined according to the method of Tom et al. [20]. Malondialdehyde (MDA) was determined using the procedure of Satoh [21]. Total proteins are determined using the method [22].

\subsection{Statistical Analysis}

Results are expressed in average \pm S.E.M. One-way analysis of variance (ANOVA) as followed by Tukey test was used for statistical evaluation. $p$ value $<0.05$ was considered as statistically significant.

\section{RESULTS}

\subsection{Phytochemistry}

The phytochemical analysis of the extract reveals the presence of saponosides, tannins, flavonoids, and but no alkaloids. The dosage of polyphenols, flavonoids, and tannins is summarized in Table 1.

\subsection{Effects Of The Extract On Glycemia Value}

Glucose has increased the glycemia's value. T. guineensis extract has decreased the glycemic rates as compared with the PC [Figure 1].

\subsection{Effects of the Extract on Blood Pressure}

After 3 weeks of treatment, the arterial blood pressure's average of rats which have received only glucose has increased significantly $(P<0.001)$. The $T$. guineensis extract has prevented this increase in arterial blood pressure's average in this model. At $400 \mathrm{mg} / \mathrm{kg}$ dosage, the extract has reduced the arterial blood pressure from $153 \pm$ $4.34 \mathrm{mmHg}$ to $126.4 \pm 2.06 \mathrm{mmHg}$. This blood pressure reduction is similar with nifédipine treatment group [Figure 2].

Table 1: Proportion of polyphenols in the extract

\begin{tabular}{lc} 
Compounds & Content \\
Total polyphenols (mg eqQ/g of extract) & 27.185 \\
Total flavonoids (mg eqQ/g of extract) & 14.29 \\
Tannins (mg eqQ/g of extract) & 10.93 \\
\hline
\end{tabular}

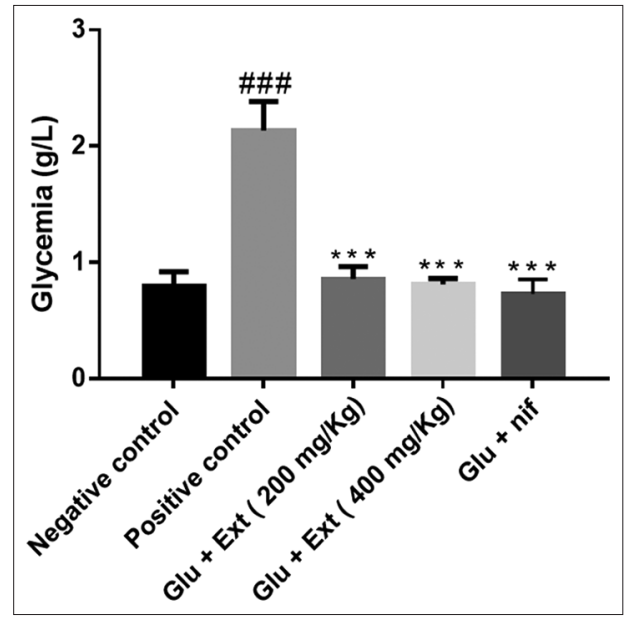

Figure 1: Effects of Trema guineensis extract on the glycemia of the rats. Every value represents the mean \pm standard error of mean, $(n=5)$. ** $P<001$ $* * * P<0.001$ significant difference in comparison with the positive control. $\# \# \# P<0.001$ significant difference in comparison with the negative control 


\subsection{Effects Of The Extract On Body Weights}

Glucose has significantly $(P<0.001)$ increased the body's weight of $\mathrm{PC}(53.00 \pm 2.98 \mathrm{~g})$ as compared to the $\mathrm{NC}(27.2 \pm 2.15 \mathrm{~g})$. There was no significant difference between weights gained in $T$. guineensis $(400 \mathrm{mg} / \mathrm{kg}$ ) glucose-treated animals as compared to NC [Figure 3].

\subsection{Effects of the Extract on Lipid Profile}

As shown in Table 2, glucose has significantly increased $(P<$ 0.001 ) the total cholesterol by $39.20 \%$, triglycerides by $82.91 \%$, atherogenic index by 242.66 , LDL-cholesterol by $324.52 \%$, and the decrease of HDL-cholesterol by $49.46 \%$, respectively, as compared to the NC. The administration of T. guineensis extracts reduced these parameters.

\subsection{Effects of the Extract on Oxidative Stress Markers}

Glucose has significantly $(P<0.001)$ increased MDA, SOD rates and reduced that of GSH and NO in aorta, heart, liver, and kidney as compared to NC. T. guineensis extract has markedly suppressed the increase of SOD, MDA and has increased the GSH and NO levels as compared to PC [Figures 4-7].

\subsection{Effects of the Extract on Liver and Kidney}

Glucose has induced a significant increased $(P<0.001)$ of urea (29.88\%), uric acid (78.98\%), GOT (102.37\%), and GTP (68.23\%), respectively, as compared to NC. T. guineensis has significantly $(P<0.001)$ reduced GOT, GTP, urea, and uric acid rates as compared to the PC. There is no difference between creatinine's rates of other groups and the control group [Table 3].

\section{DISCUSSION}

T. guineensis is used in Togo by traditional practitioners to treat many sicknesses, including cardiovascular diseases such as hypertension. In the present study, we evaluated the antihypertensive activities of $T$. guineensis extract on glucose-induced hypertension on rats. Glucose in our study has induced an increase in treated rats glycemia in relation to NC. The plant extract has significantly prevented that increase. This result can be explained by the fact that either the extract would

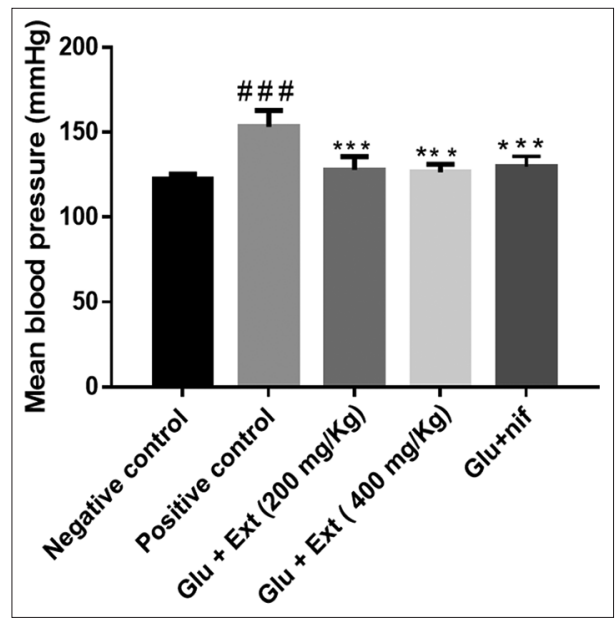

Figure 2: Effects of Trema guineensis extract on mean arterial blood pressure of the rats. Every value represents the mean \pm standard error of mean $(n=5)$. *** $P<0.001$ significant difference in comparison with the positive control. \#\# $P<0.001$ significant difference in comparison with the negative control increase insulin release to reduce the increase in blood glucose's rate or act on the liver by accelerating the formation of glycogen or on the muscles by their sensitization using glucose.

Our data have shown a significant increase in weight gain of hypertensive rats as compared to normal rats. The administration of T. guineensis extract has significantly prevented this weight gain. It

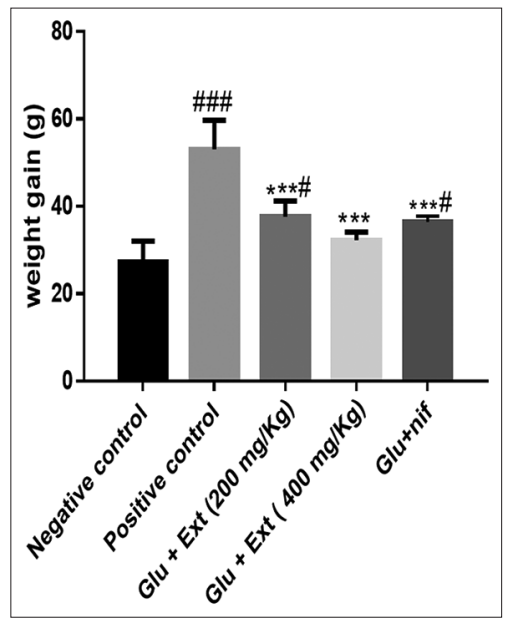

Figure 3: Effects of Trema guineensis extract on the weight of the rats. Every value represents the mean \pm standard error of mean, $(n=5) . * * * P<0.001$ significant difference in comparison with the positive control. \# $P<0.05$, \#\#\# $P<0.001$ significant difference in comparison with the negative control

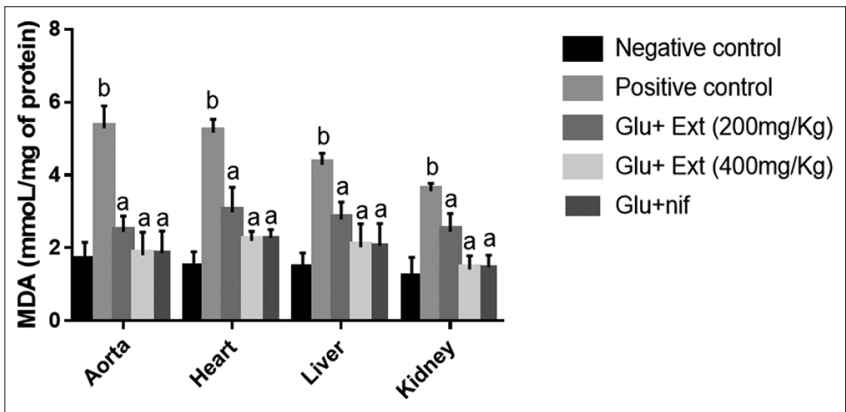

Figure 4: Effects of Trema guineensis extract on the reduced glutathione. Every value represents mean \pm standard error of mean, $(n=5)$. ${ }^{a} P<0.001$ significant difference in comparison with the positive control. ${ }^{\mathrm{b}} P<0.001$ significant difference in comparison with the negative control

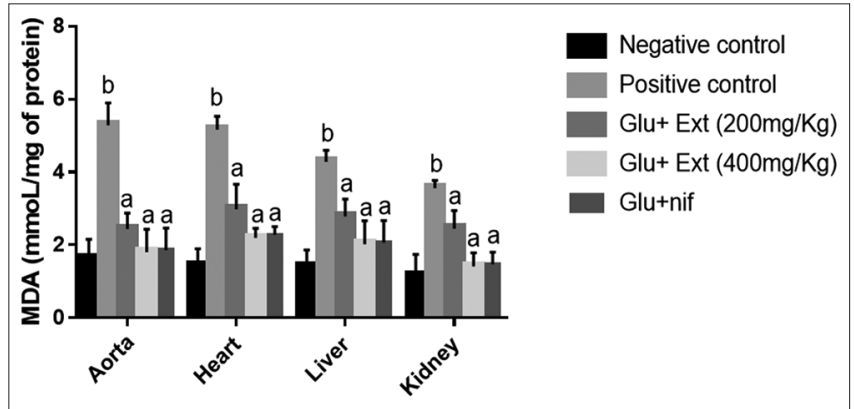

Figure 5: Effects of Trema guineensis extract on the malondialdehyde levels. Every value represents mean \pm standard error of mean, $(n=5) .{ }^{a} P<0.001$ significant difference in comparison with the positive control. ${ }^{\mathrm{b}} P<0.001$ significant difference in comparison with the negative control 
Table 2: Effects of T. guineensis extract on the lipid profile parameters of the rats

\begin{tabular}{lccccc} 
Parameters & NC & PC & Glu+Ext $(\mathbf{2 0 0} \mathbf{~ m g} / \mathbf{K g})$ & Glu+Ext (400 mg/Kg) & Glu+nif $(\mathbf{1 0} \mathbf{~ m g} / \mathbf{K g})$ \\
Triglycérides (mg/dL) & $39.80 \pm 5.28$ & $72.40 \pm 0.81 \# \#$ & $47.80 \pm 2.10^{* * *}$ & $38.20 \pm 1.56^{* * *}$ & $42.5 \pm 2.21^{* * *}$ \\
Cholesterol (mg/dL) & $55.60 \pm 3.01$ & $77.40 \pm 4.20 \# \# \#$ & $52.40 \pm 0.87 * * *$ & $52.25 \pm 0.25^{* * *}$ & $55.30 \pm 1.40^{* * *}$ \\
HDL (mg/dL) & $37.20 \pm 2.05$ & $18.80 \pm 1.98 \# \# \#$ & $37.80 \pm 1.77 * * *$ & $37.00 \pm 2.75^{* * *}$ & $25.33 \pm 3.10^{* * *}$ \\
LDL (mg/dL) & $10.44 \pm 2.76$ & $44.32 \pm 5.29 \# \# \#$ & $5.20 \pm 2.07 * * *$ & $7.56 \pm 3.16 * * *$ & $12.41 \pm 1.25^{* * *}$ \\
Indice atherogénique & $1.50 \pm 0.10$ & $5.14 \pm 0.66 \# \# \#$ & $1.39 \pm 0.084 * * *$ & $1.44 \pm 0.13^{* * *}$ & $1.88 \pm 2.21 * * *$ \\
\hline
\end{tabular}

Every value represents the mean $\pm \operatorname{ESM}$, with $(n=5)$. ${ }^{* *} P<0.001$, significant difference in comparison with the positive control. \#\#\# $P<0.001$, significant difference in comparison with the negative control, NC: Negative control, PC: Positive control, HDL: High-density lipoprotein, LDL: Low-density lipoprotein

Table 3: Effects of $T$. guineensis extract on serum parameters of liver and kidney

\begin{tabular}{lccccc} 
Parameters & NC & PC & Glu+Ext $(\mathbf{2 0 0} \mathbf{~ m g / K g})$ & Glu+Ext $(\mathbf{4 0 0} \mathbf{~ m g / K g})$ & $\mathbf{G l u}+\mathbf{n i f}(\mathbf{1 0} \mathbf{~ m g} / \mathbf{K g})$ \\
GOT (UI) & $134.80 \pm 5.15$ & $272.80 \pm 5.1 \# \# \#$ & $159.20 \pm 3.80^{* * * \# \#}$ & $149.60 \pm 2.40 * * *$ & $155.24 \pm 2.40^{* * *}$ \\
GTP (UI) & $72.40 \pm 4.47$ & $121.80 \pm 3.85 \# \# \#$ & $96.00 \pm 2.62 * *$ & $85.80 \pm 2.20 * * *$ & $73.45 \pm 1.25 * * *$ \\
Urea (mg/dL) & $41.46 \pm 1.47$ & $53.85 \pm 2.49 \# \# \#$ & $38.76 \pm 1.79 * * *$ & $36.68 \pm 2.66 * * *$ & $33.52 \pm 1.33 * * *$ \\
Uric acid (mg/L) & $14.23 \pm 0.22$ & $25.47 \pm 1.11 \# \#$ & $13.87 \pm 1.20 * * *$ & $13.62 \pm 0.92 * * *$ & $17.41 \pm 1.56^{* * *}$ \\
Creatinine (mg/dL) & $7.08 \pm 0.43$ & $6.16 \pm 0.24$ & $6.54 \pm 0.57$ & $6.96 \pm 0.20$ & $7.36 \pm 0.20$ \\
\hline
\end{tabular}

Every value represents the mean $\pm \mathrm{ESM},(n=5), * * P<0.01, * * * P<0.001$, significant difference in comparison with the positive control. \#\# $P<0.01$, \#\#\# $P<0.001$, significant difference in comparison with the negative control, GOT: Glutamic-oxaloacetic transaminase, NC: Negative control, PC: Positive control

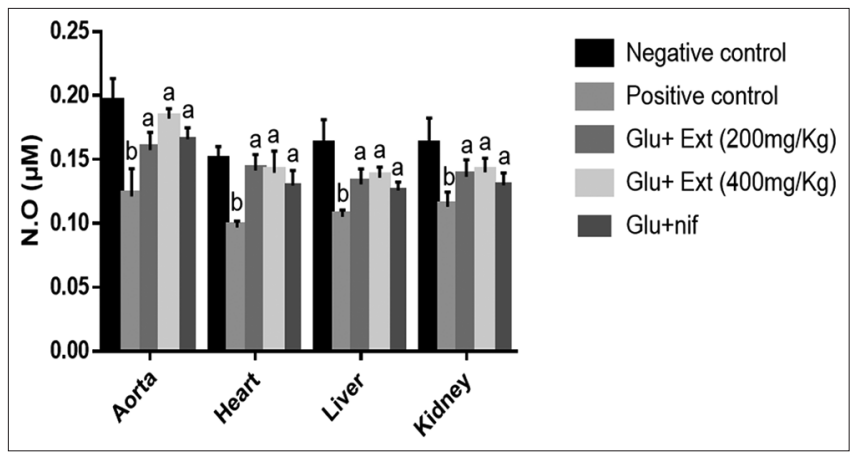

Figure 6: Effects of Trema guineensis extract on the nitric oxide of the rats. Every value represents mean \pm standard error of mean $(n=5) .{ }^{b} P<0.001$ significant difference in comparison with the positive control. ${ }^{a} P<0.001$ significant difference in comparison with the negative control

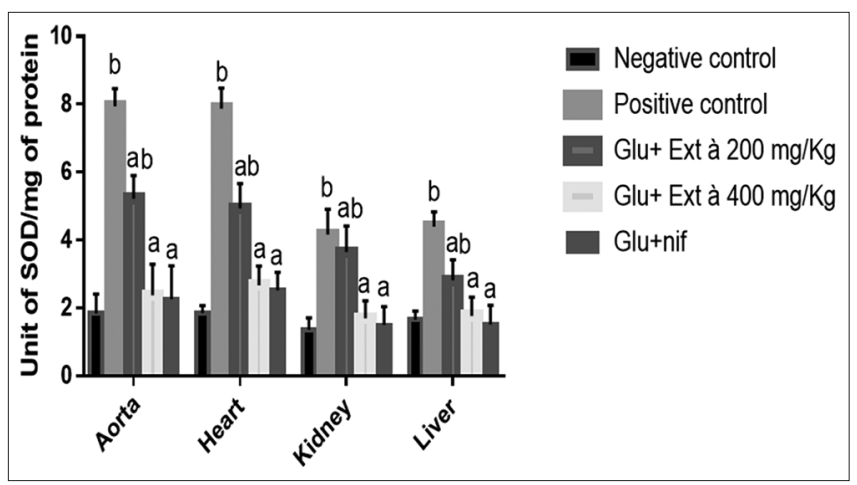

Figure 7: Effects of Trema guineensis extract on SOD levels. Every value represents mean \pm standard error of mean $(n=5) .{ }^{\mathrm{a}} P<0.001$ significant difference in comparison with the positive control. ${ }^{b} P<0.001$ significant difference in comparison with the negative control

is suggested that the chronic consumption of glucose stimulates more food intake and leads to more weight gain [23].
In our study, we have observed a significant reduction in medium arterial pressure rate to the rats treated at the same time with glucose and extract. This pressure reduction caused by the T. guineensis extract is due to the vasodilatory action of the extract, as demonstrated in vitro on the isolated aorta [15].

An increased consumption of fructose and glucose, two carbohydrates are a source of the increase in blood pressure by dyslipidemia, endothelial dysfunction, and also by the increase in oxidative stress. In the present study, glucose feeding has significantly increased total cholesterol, triglycerides, and atherogenic index. Dyslipidemia enhances vascular resistance and leads to an increase in blood pressure [24]. T. guineensis has significantly prevented total cholesterol's rise, triglycerides and atherogenic index, LDL. and increase in HDL observed in untreated glucose's rats. The vasodilator effect of the extract and its action on blood pressure decreasing may be explained by the lowering of total cholesterol, triglycerides, atherogenic index, LDL, and the increase in HDL that it causes. Furthermore, HDL increase protects heart against cardiovascular diseases risks [25]. It is suggested that the extract possesses an antihypertension effect through its antihyperlipidemic and antihypercholesterolemic's action. The increased oxidative stress and excessive formation of free radicals resulting from LDL's conversion in oxidized LDL are involved in the development of atherosclerotic lesions in the vascular endothelium [26]. This suggests that $T$. guineensis may stimulate HDL synthesis or enhance lipase protein's activity. The antihyperlipidemic's effect of the extract could represent a protective mechanism against atherosclerosis' development in rats. This suggests that lipid's improvement of a profile by the plant's extract may be responsible at least partially for arterial hypertension's prevention. Moreover, in hypertensive patients, lower concentrations of antioxidants have been documented [27]. Our inquiry is that: Does the extract of T. guineensis have its effect on oxidative stress parameters?

Cardiovascular dysfunctions in glucose-induced hypertension are the source of the increase in reactive oxygen species (ROS) and the antioxidant's alteration defense status as shown by Kasdallah-Grissa et al.[28]. Furthermore, we have measured GSH, SOD, and MDA rates 
as being the peroxidation lipid's index resulting from the activated oxygen species' reaction with membrane fatty acids [29]. The results have shown a significant increase in MDA, SOD and the decrease in GSH rates in heart, kidney, liver, and aorta. The treatment with the extract of $T$. guineensis has significantly prevented the decrease in GSH, and the increase in MDA, SOD's rates. These findings are in accordance with those found by Husain et al. [30] which have shown that glucose dose-dependently increases MDA and decreases GSH. The decrease of GSH can be explained by an increase in its use by hepatic cells, and by a decrease in the synthesis of GSH or an increase in its degradation during oxidative stress [31], because it participates in the ROS detoxification reactions [32]. It is then converted into its oxidized form (GSSG) under the GSH peroxidase's effect. This leads to GSH's consumption which is the reason for its decrease in PC rats [33]. Therefore, the extract favors GSH's synthesis and stops its degradation by fighting against the oxidative stress. Thus, treatment with plant's extract has shown antioxidant properties by preventing tissues' lipid peroxidation and increasing GSH. The increase in SOD is due to high oxidative stress causing the increase in this enzyme's production to fight against the stress. The reduction observed in SOD antioxidant enzyme's concentrations in all extract's treated groups may occur due to an oxidative stress status' total reduction. Our results suggest that the extract induces an antioxidant effect, which may due to this plant richness in bioactive compounds and antioxidant that inhibit the lipoperoxidation and alteration caused by the excessive production of free radicals as shown by Liu et al. [34].

In the current study, glucose has significantly decreased the NO. Plant extract, given simultaneously with glucose, has prevented the decrease in NO. The extract has corrected this deficit in NO's rate. It suggests that the extract could prevent the endothelial dysfunction caused by NO's deficit.

We have evaluated the glucose's toxicity on liver and kidney's functions by assessing transaminases (GOT and GTP), uric acid, and urea's rate. The hepatic injury induced by glucose has resulted in an increase in serum GOT and GTP rates due to cellular enzymes' leakage in the circulation. T. guineensis has inhibited the raising in GOT, GTP serum's rates and without reducing them to normal control values; this is similar with the effect on oxidative stress markers. This may indicate that oxidative stress is involved in hepatotoxicity's mechanisms due to glucose chronic consumption. This reduction in transaminases can also be explained by the gluconeogenesis reduction's process; an essential step requiring the GOT and GTP's intervention [35]. Serum enzymes' low rate after the concomitant treatment with $T$. guineensis as compared to NC suggests liver and kidney's protective effects of the extract.

Phytochemical studies of the extract have revealed polyphenols' presence such as flavonoids and tannins. The main characteristic of polyphenols is that they are very powerful antioxidants [36]. Indeed, flavonoids are also known to have a preventive role against cardiotoxicity, their inhibition of lipid peroxidation and their ability to prevent various hematological disorders [37]. Indeed, flavonoids provide protection against free radicals by preventing their binding with cell membrane lipids; which results in a decrease in MDA (lipid peroxide) and the hematological composition's protection by allowing good erythrocyte regeneration and prevention against leukopenia and thrombocytopenia observed in the presence of free radicals [38]. Flavonoids have an ability to capture and deactivate free radicals. Flavonoids are known for their protective effect on cardiovascular health by modifying several pathological processes involved in cardiovascular development diseases by inhibition of the oxidation of LDL cholesterol (bad cholesterol) by free radicals. These results obtained during our experiment would be due to the polyphenols contained in the extract and in particular the flavonoids.

\section{CONCLUSION}

The side effects of chronic administration of glucose have been established in this study, and we can suggest lipid accumulation, oxidative stress and tissue damage as a bridge linking the high consumption of sugar to arterial hypertension. These results scientifically confirm the antihypertensive properties of the extract of T. guineensis as used in Togolese traditional medicine and suggest that this effect may result from its blood pressure or lipid-lowering and its antioxidant or vasodilator effects.

\section{ACKNOWLEDGMENT}

Nil.

\section{REFERENCES}

1. Johnson OE, Adedoyin RA, Awotidebe TO, Mbada CE, Otolorin OA, Owoseni I. Cardiovascular risk among under graduates in a Nigerian university. Int J Public Health Epidemiol 2013;2:85-9.

2. de Kloet AD, Krause EG, Shi PD, Zubcevic J, Raizada MK, Sumners C, et al. Neuroimmune communication in hypertension and obesity: A new therapeutic angle? Pharmacol Ther 2013;138:428-40.

3. Okumura N, Kondo T, Matsushita K, Osugi S, Shimokata K, Matsudaira $\mathrm{K}$, et al. Associations of proteinuria and the estimated glomerular filtration rate with incident hypertension in young to middle-aged Japanese males. Prev Med 2014;60:48-54.

4. Fourcade L, Paul P, Mafart B. Arterial hypertension in sub-Saharan Africa. Nitric oxide production and inhibits angiogenesis. Circulation 2007;106:913-9.

5. Taylor DA, Abdel-Rahman AA. Novel strategies and targets for the management of hypertension. Adv Pharmacol 2009;57:291-345.

6. Hernández EA, Segura-Cobos D, López-Sánchez P. Plants present in Mexico with studies in metabolic syndrome. J Med Plants Stud 2016;4:95-103.

7. Hwang IS, Ho H, Hoffman BB, Reaven GM. Fructose-induced insulin resistance and hypertension in rats. Hypertension 1987;10:512-6.

8. El Midaoui A, De Champlain J. Prevention of hypertension, insulin resistance, and oxidative stress by $\alpha$-lipoic acid. Hypertension 2002; 39: 303-307.

9. Reaven GM, Ho H. Sugar-induced hypertension in sprague-dawley rats. Am J Hypertens 1991;4:610-4.

10. Baum M. Insulin stimulates volume absorption in the rabbit proximal convoluted tubule. J Clin Invest 1987;79:1104-9.

11. Buñag $\mathrm{RD}$, Tomita $\mathrm{T}$, Sasaki $\mathrm{S}$. Chronic sucrose ingestion induces mild hypertension and tachycardia in rats. Hypertension 1983;5:218-25.

12. Tomiyama H, Kimura Y, Okazaki R, Kushiro T, Abe M, Kuwabara Y, et al. Close relationship of abnormal glucose tolerance with endothelial dysfunction in hypertension. Hypertension 2000;36:245-9.

13. Cai H, Harrison DG. Endothelial dysfunction in cardiovascular diseases: The role of oxidant stress. Circ Res 2000;87:840-4.

14. Ahyi MA, Ake AL, Akpagana K, Chibon P, EL-Hadji Watara A, Eyme M, et al. Contribution aux Études Ethnobotaniques et Floristiques du Togo. Paris: Rapport de l'ACCT; 1986.

15. Kadissoli B, Mouzou AP, Tekou E, Aklikokou A, Gbeassor M. Effect of the semi-ethanolic extract of the leaves of Trema guineensis (Celtidacea) on the smooth musculature of the Wistar rats. Int J Biol Chem Sci 2012;6:985-94.

16. Odebiyi OO, Sofowora EA. Phytochemical screening of Nigerian 
medicinal plants II. Lloydia 1978;41:234-46.

17. Mtopi OS, Dimo T, Nguelefack TB, Djomeni PD, Rakotonirina SV, Kamtchouing P. Effects of Brillantaisia nitens Lindau (Acanthaceae) methylene chloride/methanol leaf extract on rat arterial blood pressure and heart rate. Pharmacol Online 2007;1:495-510.

18. Sedlak J, Lindsay RH. Estimation of total, protein-bound, and nonprotein sulfhydryl groups in tissue with Ellman's reagent. Anal Biochem 1968;25:192-205.

19. Misra HP, Fridovich I. The role of superoxide anion in the autoxidation of epinephrine and a simple assay for superoxide dismutase. J Biol Chem 1972;247:3170-5.

20. Tom EN, Demougeot C, Mtopi OB, Dimo T, Djomeni PD, Bilanda DC, et al. The aqueous extract of Terminalia superba (Combretaceae) prevents glucose-induced hypertension in rats. J Ethnopharmacol 2011;133:828-33.

21. Satoh K. Serum lipid peroxide in cerebrovascular disorders determined by a new colorimetric method. Clin Chim Acta 1978;90:37-43.

22. Bradford MM. A rapid and sensitive method for the quantitation of microgram quantities of protein utilizing the principle of protein-dye binding. Anal Biochem 1976;72:248-54.

23. Johnson RJ, Segal MS, Sautin Y, Nakagawa T, Feig DI, Kang DH, et al. Potential role of sugar (fructose) in the epidemic of hypertension, obesity and the metabolic syndrome, diabetes, kidney disease, and cardiovascular disease. Am J Clin Nutr 2007;86:899-906.

24. Stevens RJ, Coleman RL, Shine BL, Holman RR. Could nonHDL cholesterol replace total/HDL cholesterol ratio to estimate coronary heart disease risk in the UKPDS risk engine? Diabetologia 2004;47:A61.

25. Adi K, Metowogo K, Mouzou A, Lawson P, Eklu-Gadegbeku K, Agbonon A, et al. Evaluation of cardioprotective effects of Parkia biglobosa (Jacq. Benth) mimosaceae sterm back. J Appl Pharm Sci 2013;3:60-64.

26. Pasupathi P, Saravanan G, Farook J. Oxidative stress bio markers and antioxidant status in cigarette smokers compared to nonsmokers. J Pharm Sci Res 2009;2:55-62.

27. Rodrigo R, Prat H, Passalacqua W, Araya J, Guichard C, Bächler JP, et al. Relationship between oxidative stress and essential hypertension. Hypertens Res 2007;30:1159-67.

28. Kasdallah-Grissa A, Mornagui B, Aouani E, Hammami M, Gharbi N, Kamoun A, et al. Protective effect of resveratrol on ethanol-induced lipid peroxidation in rats. Alcohol Alcohol 2006;41:236-9.

29. Nayeemunisa A, Kumuda MR. Cardioprotective effects of Cichorium intybus in ageing myocardium of albino rats. Curr Sci 2003;84:94-43.

30. Husain K, Mejia J, Lalla J, Kazim S. Dose response of alcoholinduced changes in $\mathrm{BP}$, nitric oxide and antioxidants in rat plasma. Pharmacol Res 2005;51:337-43.

31. Loven D, Schedl H, Wilson H, Daabees TT, Stegink LD, Diekus M, et al. Effect of insulin and oral glutathione on glutathione levels and superoxide dismutase activities in organs of rats with streptozocininduced diabetes. Diabetes 1986;35:503-7.

32. Abou-Seif MA, Youssef AA. Evaluation of some biochemical changes in diabetic patients. Clin Chim Acta 2004;346:161-70.

33. Baynes JW, Thorpe SR. Role of oxidative stress in diabetic complications: A new perspective on an Old Paradigm. Diabetes 1999;48:1-9.

34. Liu L, Howe P, Zhou YF, Xu ZQ, Hocart C, Zhan R, et al. Fatty acids and beta-carotene in Australian purslane (Portulaca oleracea) varieties. J Chromatogr A 2000;893:207-13.

35. Derouiche S, Kechrid Z. Influence of calcium supplements on zinc status, carbohydrate metabolism and the liver activity of detoxifying glutathione enzymatic system in alloxan-induced diabetic rats. J Exp Biol Agric Sci 2013;1:425-9.

36. Oszmianski J, Wojdylo A, Lamer-Zarawska E, Swiader K. Antioxidant tannins from Rosaceae plant roots. Food Chem 2007;100:579-83.

37. Sadzuka Y, Sugiyama T, Shimoi K, Kinae N, Hirota S. Protective effect of flavonoids on doxorubicin-induced cardiotoxicity. Toxicol Lett 1997;92:1-7.

38. Rao CV, Vijayakumar M. Effect of quercetin, flavonoids and alpha-tocopherol, an antioxidant vitamin, on experimental reflux oesophagitis in rats. Eur J Pharmacol 2008;589:233-8.

\footnotetext{
How to cite this article:

Kadissoli B, Mouzou PA, Pakoussi T, Eklu-Gadegbeku K, Aklikokou AK,

Gbeassor M. Effect of Trema guineensis leaves (celtidaceae) on glucose-induced

hypertension in Wistar rats. 2019;7(02):42-47. DOI: 10.7324/JABB.2019.70208
} 